\title{
Saucerization versus dissection on the repair of vesicovaginal fistulae
}

\author{
ANIEFIOK J. UMOIYOHO ${ }^{1, A, B, D-F}$, OLUJIMI ABIONA OLATUNBOSUN², c-F \\ ${ }^{1}$ Department of Obstetrics and Gynecology, Faculty of Clinical Sciences, College of Medical Sciences, \\ University of Uyo, Akwa Ibom State, Nigeria \\ ${ }^{2}$ Department of Obstetrics and Gynecology, University of Uyo Teaching Hospital, Uyo Akwa Ibom State, Nigeria
}

A - Study Design, B - Data Collection, C - Statistical Analysis, D - Data Interpretation, E - Manuscript Preparation, F - Literature Search, G - Funds Collection

Summary Background. An obstetrics fistula is both an unfortunate and annoying preventable complication that follows poorly managed prolonged obstructed labor. Despite an improvement in obstetric management, this condition has remained with us in developing countries partly because of the failure of effective maternity care, as well as lack of adequately trained surgeons to repair the backlog of cases.

Objectives. This study was therefore designed to determine whether a simpler and quicker method of fistula repair - saucerization - could be recommended for fistula repair by relatively inexperienced surgeons as compared to the more technical dissection method, which requires a higher level of surgical expertise.

Material and methods. This was an intervention analytical study of 77 fistula repairs done between 2010 and 2013 using either saucerization or dissection methods. The success (closure) rate for each method was calculated. A test of association between surgical technique and outcome of surgery using the chi-squared test was done. Residual fistulae dimensions and degree of scarring were assessed and analyzed using SPSS version 20.

Results. The dissection method had a higher, though not statistically significant, cure rate than the saucerization method $(92.3 \%$ vs $76.3 \%, p=0.053)$. Residual fistulae dimensions were larger in the saucerization group, while fibrosis was higher in the dissection method.

Conclusions. The saucerization technique for the repair of VVF was inferior to the dissection method, although the difference in cure rate was not statistically significant.

Key words: dissection, obstetric fistula, vesicovaginal fistula.

Umoiyoho AJ, Olatunbosun OA. Saucerization versus dissection on the repair of vesicovaginal fistulae. Fam Med Prim Care Rev 2017; 19(4): 408-411, doi: https://doi.org/10.5114/fmpcr.2017.70817.

\section{Background}

A vesicovaginal fistula (VVF), especially an obstetric fistula, is an obstetric calamity prevalent only in developing countries. It is a major public health problem in Nigeria, a reminder of gender inequality and a reflection on the poor state of the health care system in affected countries.

An obstetric fistula is an abnormal connection between the genital tract and the urinary bladder and/or rectum arising from prolonged obstructed labor [1], leading to continuous and uncontrolled leakage of urine, often as a result of poor or insufficient emergency obstetric care and a skilled birth attendant $[2,3]$.

Those women affected live with persistent urinary and/or fecal incontinence, which, with the accompany stench, leads to social isolation, divorce, depression and even abuse. They lose their self-esteem and are ostracized from their home and society [4].

An estimated 2 million women are said to be living with untreated obstetric fistulae globally, while approximately 50,000$-100,000$ new cases of fistulae occur annually [5]. Unfortunately, most of these cases occur in developing countries, especially Sub-Saharan African and parts of Asia [5, 6].

In 2008, the Nigeria demographic health survey put the prevalence of obstetric fistulae at $0.5 \%$ in South-South Nigeria, a value higher than the national average of $0.4 \%$ [7]. A total of
$400,000-500,000$ cases of VVF are estimated to be in Nigeria, with about 20,000 new cases occurring every year [8]. The unmet need for fistula repair has been estimated to be as high as 99\% [4], and most women/girls with fistulae have been predicted to die without receiving treatment [9]. According to a study entitled: survey and needs assessment, approximately 2,000$-4,000$ fistula repair surgeries are carried out yearly in Nigeria [5]. Based on this rate of repair, it has been estimated that it will take 100 years to deal with the backlog of cases, ignoring new cases [8]. This is also against the backdrop that only about 33 surgeons provide fistula repair services in Nigeria [10]. The surgical closure rate of vesicovaginal fistulae has been reported to be as high as $90 \%$, though this rate varies from one repair hospital to another $[2,11]$. The successful repair of a fistula depends on the experience of the surgeon, as well as the use of an appropriate technique. Other factors are fistula site, number, size and degree of scarring, as well as number of previous attempts at repair and post-operative care [12]. The first author of this study, in his earlier work, had developed a prognostic scoring system that helped with patient selection, an important factor that influences the surgical success rate [13].

Surgical management of fistulae is either through the abdominal or vaginal route. The preferred route of repair of obstetric VVF for most fistula surgeons in these setting is the vaginal approach [14]. The vaginal route offers the advantage of avoidance of laparotomy, thus preventing bowel manipulation, shorter recovery time, lesser morbidity, blood loss and 
postoperative bladder irritability, as well as less postoperative pain [15].

Transvaginal repair of VVF involves two main surgical techniques - the saucerization and dissection method. Saucerization is used for small and residual fistulae. It offers the advantage of simplicity. It avoids a difficult layer dissection and produces very little vaginal shortening [16]. Despite these advantages, saucerization has the disadvantage of having less success, especially for fistulae with fibrosis.

\section{Objectives}

This study was carried out to assess the success rate achievable using these two different techniques and to see if saucerization can be recommended for surgeons with less experience.

\section{Material and methods}

\section{Study design}

This was an interventional analytical study of VVF repairs done by the first author. Seventy-seven obstetric fistula patients were randomly assigned to either the saucerization or dissection group. The two groups were matched for age, parity, duration of fistula and size of fistula.

\section{Study setting}

The study was carried out at the Family Life Center, Mbribit Itam in Akwa Ibom State, Nigeria, between January 2010 and December 2013. The Family Life Center is the Medical Missionary of Mary Mission Hospital, located at the outskirt of Uyo. It serves as a referral center for VVF cases in the state, as well as the neighboring states of Abia, Cross River, Rivers, Imo and Benue.

\section{Participants}

Ethical approval was obtained from the institution review board of the hospital, with registration number IRB/13/17. Cases were selected from among fistula patients that presented for fistula surgery during the study period. The cases were randomly assigned to the two groups (saucerization and dissection) by balloting. The first patient in each pair was assigned to either of the two groups by balloting, while the second patient was placed in the other group. Cases were selected using the prognostic scoring system [13]. A score of $\leq 4$ means a simple VVF. The inclusion criteria included a small size fistulae $<4 \mathrm{~cm}$, midvaginal fistulae and minimal scarring.

The exclusion criteria included juxtacervical (high) fistulae, because of the difficulty in saucerizing such fistulae, fistulae $>4$ $\mathrm{cm}$, juxtaurethral fistulae and fistulae where the ureteric orifice is less than $2 \mathrm{~cm}$ from the fistula margin.

\section{Variables}

The outcome measures for this study were:

1) the success rate (restoration of continence), i.e. closure of the fistula,

2) the dimension of residual fistulae at 3 months using calipers and a ruler,

3) subjective assessment of the degree of scarring by the first author.

The exposure variables were the two surgical techniques used during the study. The techniques of repair were the saucerization and dissection methods. The saucerization method is useful for small fistulae. It does not involve the separation of the vaginal layer from the underlying bladder wall. The edges of the fistula are trimmed off obliquely, stopping short of the blad- der mucosa, thus converting the cylindrical track into a shallow saucer. The defect is then closed in two layers. The first row is placed in the bladder wall just superficial to the mucosa. This is followed by a second row in the vaginal wall [16]. The dissection method, on the other hand, entails dissecting the vaginal wall off the underlying bladder so that the bladder wall around the fistula can be mobilized and drawn across the defect. The bladder wall is repaired in two layers. Potential confounders include stress urinary incontinence and detrusor instability. These were ruled out through detailed history taking and examination of the patients.

\section{Data sources/measurement}

The variables measured were the age and parity of the patients. These were obtained from their case notes. The outcome variable (closure of fistula) was determined at 3 months, from history of whether or not the patient still leaks urine and during vaginal examination to rule out stress incontinence and detrusor instability. If a residual fistula is diagnosed, the widest dimension is measured using a ruler and a subjective assessment of the degree of fibrosis noted. The measurement and subjective assessment were done for both groups using the same instrument and by the same person to allow for comparability of measurements.

\section{Bias}

The potential for bias during selection of cases to either the dissection or saucerization group was minimized by randomly selecting them through balloting. The surgeries, either by the dissection or saucerization methods, were also carried out by the same surgeon, thus removing the bias that would have arose if surgeons with different surgical skills had done the operations.

\section{Study size}

The sample size was determined by the number of eligible fistula patients that presented during the study period.

\section{Quantitative variables}

These included age and parity of the patients and the sizes of the residual fistulae. Because of the wide spread of the ages and for ease of data management and analysis, the ages were grouped into six categories with a class width of five. Parity and residual fistula size were analyzed ungrouped.

\section{Statistical methods}

The data generated was analyzed using SPSS version 20, and the results were expressed in numbers and percentages. Descriptive analysis was performed using means, percentages and ranges. The variables (age, parity and residual fistula size) were checked for normal distribution using a combination of statistical tests, which included the Kolmogorov-Smirnov test, skewness, kurtosis and their critical values. These tests show that these variables are normally distributed. The chi-squared test was used to test for any association between categorical variables, such as surgical technique and outcome of repair. Association was considered significant at a $p$-value of less than 0.05 . A paired sample $t$-test for the mean difference of fistula sizes before repair and post-repair were also carries out.

\section{Results}

\section{Participants}

During the period under study, 123 patients with incontinence presented for surgery. History taking and examination 
revealed that only 115 of the women had fistulae, while 8 had a combination of stress incontinence and detrusor instability. Of the 115 patients examined for eligibility, 77 were eligible and were included in the study and analysis. All participants in the study presented for a follow up.

\section{Descriptive data}

Table 1 shows the demographic characteristics of the patients. The age, parity and residual fistula size of the patients shows normal distribution. The age ranges of the women were 17 to 36 years. Their mean ages were: 25.4 years (SD 5.8) and 25.6 years (SD 5.2) for the saucerization and dissection groups, respectively, while the average parity was 3.5 (SD 1.7) and 3.3 (SD 1.7), respectively. A majority of the women were married.

\begin{tabular}{|l|l|l|l|l|}
\hline \multicolumn{5}{|l|}{ Table 1. Demographic characteristics of VVFatients } \\
\hline Age & Saucerization & & Dissection & \\
\hline Years & $n$ & $\%$ & $n$ & $\%$ \\
\hline$<15$ & 0 & 0.0 & 0 & 0.0 \\
\hline $15-20$ & 8 & 21.0 & 6 & 15.4 \\
\hline $21-25$ & 10 & 26.3 & 12 & 30.8 \\
\hline $26-30$ & 12 & 31.6 & 13 & 33.3 \\
\hline $31-35$ & 5 & 13.2 & 6 & 15.4 \\
\hline$>35$ & 3 & 7.9 & 2 & 5.1 \\
\hline Total & 38 & 100.0 & 39 & 100.0 \\
\hline Parity & $n$ & $\%$ & $n$ & $\%$ \\
\hline 1 & 7 & 18.4 & 9 & 23.1 \\
\hline 2 & 2 & 5.3 & 4 & 10.3 \\
\hline 3 & 10 & 26.3 & 7 & 17.9 \\
\hline 4 & 11 & 28.9 & 9 & 23.1 \\
\hline 5 & 3 & 7.9 & 6 & 15.4 \\
\hline$>5$ & 5 & 13.2 & 4 & 10.3 \\
\hline Total & 38 & 100.0 & 39 & 100.0 \\
\hline $\begin{array}{l}\text { Marital } \\
\text { Status }\end{array}$ & $\boldsymbol{n}$ & $\%$ & $n$ & $\%$ \\
\hline Married & 34 & 89.5 & 36 & 92.3 \\
\hline Single & 4 & 10.5 & 3 & 7.7 \\
\hline Total & $\mathbf{3 8}$ & $\mathbf{1 0 0 . 0}$ & $\mathbf{3 9}$ & $\mathbf{1 0 0 . 0}$ \\
\hline & & & & \\
\hline
\end{tabular}

\section{Outcome data}

Of the 38 women that had saucerization of their VVF, 29 (76.3\%) had successful closure of the fistula, while $9(23.7 \%)$ had failed closure. On the other hand, of the 39 women in the dissection group, 36 (92.3\%) women had restoration of continence, while only $3(7.7 \%)$ had failed closure, as shown in Table 2 . The test of association between surgical technique and outcome of surgery, done using the chi-squared test, was, however, not significant: $\chi^{2}=3.74, d f=1, p=0.053$.

\begin{tabular}{|l|l|l|}
\hline \multicolumn{3}{|l|}{ Table 2. Outcome of fistula repairs } \\
\hline & $\begin{array}{l}\text { Saucerization } \\
n(\%)\end{array}$ & $\begin{array}{l}\text { Dissection } \\
n(\%)\end{array}$ \\
\hline Success & $29(76.3)$ & $36(92.3)$ \\
\hline Failure & $9(23.7)$ & $3(7.7)$ \\
\hline Total & $\mathbf{3 8}$ & $\mathbf{3 9}$ \\
\hline
\end{tabular}

A review of the 12 failed cases for assessment of residual fistula sizes and the degree of scarring done at 3 months following repair revealed that the residual fistulae were of a larger dimension than the pre-surgery dimension in the saucerization group, unlike in the dissection group, as shown in Table 3. The mean residual fistula size in the saucerization group was 3.3 (SD 0.8 ), while for the dissection group, it was 1.7 (SD 0.2).

\begin{tabular}{|l|l|l|}
\hline \multicolumn{2}{|l|}{ Table 3. Residual fistulae sizes } \\
\hline \multirow{5}{*}{ Saucerization } & Pre-surgery $(\mathrm{cm})$ & Post-surgery $(\mathrm{cm})$ \\
\hline & 3.0 & 3.5 \\
\cline { 2 - 3 } & 3.4 & 3.8 \\
\cline { 2 - 3 } & 3.7 & 4.1 \\
\cline { 2 - 3 } & 4.0 & 4.5 \\
\cline { 2 - 3 } & 2.0 & 3.0 \\
\cline { 2 - 3 } & 2.5 & 3.1 \\
\cline { 2 - 3 } & 3.0 & 3.3 \\
\cline { 2 - 3 } & 0.5 & 2.0 \\
\cline { 2 - 3 } & 1.0 & 2.5 \\
\hline Dissection & 3.0 & 1.5 \\
\cline { 2 - 3 } & 3.5 & 1.7 \\
\cline { 2 - 3 } & 4.0 & 1.9 \\
\hline
\end{tabular}

\section{Main results}

The paired sample $t$-test showed a statistically significant effect from surgery $(t(8)=-4.732, p<0.05$,) with an increase in the size of fistulae from pre-saucerization to post-saucerization (mean difference $=0.7444,95 \% \mathrm{Cl}=0.3816-1.1073$ ) . Likewise, in the dissection group, there was a statistically significant effect from surgery $(t(2)=10.392, p<0.05)$, with a decrease in the sizes of fistulae from pre-dissection to post-dissection (mean difference $=1.8000,95 \% \mathrm{Cl}=1.0548-2.5452$ ). Also, fibrosis (scarring) were found in 7 (77.8\%) of the 9 failed cases done by saucerization and in all the $3(100 \%)$ failed cases in the dissection group. The fibrosis was described as moderate to severe.

\section{Discussion}

\section{Key results}

This study revealed a slightly higher success rate for the dissection method over the saucerization method. The difference, however, was not statistically significant. There was a significant increase in the sizes of residual fistulae for failed cases in the saucerization group, with associated moderate/severe fibrosis.

\section{Interpretation}

The WHO proposed a successful closure rate for first repair at $85 \%$ in each facility and a continence rate of $90 \%$ [17]. Different authors in developing countries have reported a primary fistula closure rate of 61-95\% [18]. However, these are largely dissection methods. The closure rate using the dissection method in this study is similar to other studies $[17,18]$. A search of published literature on comparison of the saucerization versus dissection technique did not yield any publications. This is the first time this type of study has been carried out to our knowledge. Therefore, it may be reasonable to encourage relatively inexperienced surgeons to use this method (while learning the dissection method) due to the added advantages, which include short operative time, absence of difficult dissection, simplicity and less bleeding [16]. It must be stated, however, that the saucerization method is not suitable for large defects, or when the tissues are not mobile [16]. Prior repair of fistulae, among other factors, have also been shown to be an independent prognostic factor for fistula closure [19]. In spite of the above advantages of the saucerization method, the significant increase in the sizes of residual fistulae for failed cases in the saucerization group, with 
the associated moderate/severe fibrosis, are negative prognostic factors for subsequent repair, as supported by other studies [19-21] that showed that both severe vaginal scarring and large fistulae that required extensive dissection to be able to close the defect with the associated postoperative scarring are risk factors for a poor surgical outcome.

\section{Generalizability}

The applicability of the findings to the general population is limited due to the small sample size in each group and the need for a similar study in different settings. There is also a need for a more objective method of assessing the degree of fibrosis.

\section{Limitations of the study}

The subjective assessment of fibrosis may have caused some imprecision in the assessment of the degree of fibrosis.

\section{Conclusions}

The success rate of VVF repair via the saucerization technique is inferior to that of the dissection technique, even though the difference was not statistically significant. Although the saucerization technique is technically easier to perform, the attendant higher failure rate and resultant postoperative increase in size of residual fistulae does not justify its use, especially since the number of failed attempts at repair adversely affect the prognosis.

Source of funding: This work was funded by the authors' own resources.

Conflict of interest: The authors declare no conflict of interests.

\section{References}

1. Lewis G, de Bernis L, eds. Obstetric fistula, guiding principle for clinical management and programme development. WHO; $2007: 1-73$.

2. Tayler-Smith K, Zachariah R, Manzi M, et al. Obstetric fistula in Burundi: a comprehensive approach to managing women with this neglected disease. BMC Pregnancy Childbirth 2014; 14: 164, doi: https://doi.org/10.1186/1471-2393-13-164.

3. Osotimehin B. Obstetric fistula: ending the health and human rights tragedy. Lancet 2013; 381(9879): 1702-1703.

4. Arrowsmith SD, Ruminjo J, Landry EG. Current practices in treatment of female genital fistula: a cross sectional study. BMC Pregnancy and Childbirth 2010; 10: 73, doi: 10.1186/1471-2393-10-73.

5. Federal Government of Nigeria. Federal Ministry of Health, Department of Family Health, Reproductive Health Division. National Strategic Framework for the elimination of obstetric fistula in Nigeria, 2011-2015. 2012: 1-64.

6. Tebeu PM, Fomulu JN, Khaddaj S, et al. Risk factors for obstetric fistula: a clinical review. Int Urogynecol J 2012; 23(4): 387-394.

7. Nigeria Demographic and Health Survey. 2008: 139-141.

8. Federal Government of Nigeria. Federal Ministry of Health, Department of Family Health, Reproductive Health Division. Standard of practice on obstetric fistula in Nigeria. 2011: 1-44.

9. United Nations General Assemby, A/71/306. Intensifying efforts to end obstetric fistula. 2016: 1-19.

10. United Nations Population Fund (UNFPA), Engender Health. Obstetric fistula needs assessment; findings from 9 African countries. 2003: 57-76.

11. Delamou A, Diallo M, Beavogui AH, et al. Good clinical outcomes from a 7 year holistic programme of fistula repair in Guinea. Trop Med Int Health 2015; 20(6): 813-819.

12. Kayondo M, Wasswa S, Kabakyenga J, et al. Predictor and outcome of surgical repair of obstetric fistula at a regional referral hospital, Mbarara, Western Uganda. BMC Urology 2011; 11(23): 1-9, doi: https://doi.org/10.1186/1471-2490-11-23.

13. Umoiyoho AJ, Inyaang-Etoh EC. Obstetric fistula repair: a guide to patient selection. Urogynaecolgia 2011; 25(e8): 28-30.

14. Stanford E, Romanzi L. Vesicovaginal fistula: what is the preferred closure technique? Int Urogynecol J 2012; 23(4): 383-385.

15. Kumar S, Kekre NS, Gopalakrishnann G. Vesicovaginal fistula: an update. Indian J Urol 2007; 23(2): 187-191.

16. Lawson JB. Injuries of the urinary tract. In: Lawson JB, Stewart DB, eds. Obstetric and gynaecology in the tropics and developing countries. London: Edward Arnold (Publishers) Ltd.; 1970; 481-522.

17. de Bernis L. Obstetric fistula: guiding principles for clinical management and programme development: a new WHO guideline. Int J Gynaecol Obstet 2007; 99(1): 5117-5121.

18. Macks GR, Roth TM. Vesicovaginal fistula and urethrovaginal fistula. In: Rock JA, Jone III HV, eds. Te Linde's operative gynecology. $10^{\text {th }}$ ed. Philadelphia: Lippincott Williams \& Wilkins; 2008: 973-982.

19. Mark AB, Frajzyngier V, Ruminjoet J, et al. Determinants of postoperative outcomes of female genital fistula repair surgery. Obstet Gynecol 2012; 120(3): 524-531.

20. Browning A. Risk factors for developing residual urinary incontinence after obstetric fistula repair. BJOG 2006; 113(4): 482-485.

21. McFadden E, Taleski SJ, Bocking A, et al. Retrospective review of predisposing factors and surgical outcomes in obstetric fistula patients at a single teaching hospital in Western Kenya. J Obstet Gynaecol Can 2011; 33(1): 30- 35.

Tables: 3

Figures: 0

References: 21

Received: 26.11.2016

Revised: 14.02.2017

Accepted: 31.03.2017

Address for correspondence:

Olujimi Abiona Olatunbosun, MD

Department of Obstetrics and Gynaecology

University of Uyo Teaching Hospital

Uyo, Akwa Ibom, Nigeria

Tel.: 08033704583

E-mail: drsofoluwe@yahoo.com 